\title{
A comparative study of density functional and density functional tight binding calculations of defects in graphene
}

\author{
Alberto Zobelli, ${ }^{1, *}$ Viktoria Ivanovskaya, ${ }^{2}$ Philipp Wagner, ${ }^{2}$ Irene Suarez-Martinez, ${ }^{3}$ Abu Yaya, ${ }^{2}$ and Chris P. Ewels ${ }^{2}$ \\ ${ }^{1}$ Laboratoire de Physique des Solides, Univ. Paris-Sud, CNRS-UMR 8502, 91405, Orsay, France \\ ${ }^{2}$ Institut des Matériaux Jean Rouxel (IMN), CNRS UMR 6502, University of Nantes, 44322 Nantes, France \\ ${ }^{3}$ Nanochemistry Research Institute, Curtin University of Technology, Perth, Western Australia 6845, Australia
}

The density functional tight binding approach (DFTB) is well adapted for the study of point and line defects in graphene based systems. After briefly reviewing the use of DFTB in this area, we present a comparative study of defect structures, energies and dynamics between DFTB results obtained using the dftb + code, and density functional results using the localised Gaussian orbital code, AIMPRO. DFTB accurately reproduces structures and energies for a range of point defect structures such as vacancies and Stone-Wales defects in graphene, as well as various unfunctionalised and hydroxylated graphene sheet edges. Migration barriers for the vacancy and Stone-Wales defect formation barriers are accurately reproduced using a nudged elastic band approach. Finally we explore the potential for dynamic defect simulations using DFTB, taking as an example electron irradiation damage in graphene.

\section{INTRODUCTION}

The family of carbon structures is much larger than its most notable components and the number of new members synthesized each year makes it hard to categorize all carbon forms. 1 Our ability to describe computationally the structure of realistic carbon systems faces the additional difficulty represented by the presence of native defects, which often dominate the mechanical, electronic and chemical properties of their host material. Furthermore, defect combinations can also serve as elemental topological transformations that when applied to original perfect forms generate more complex structures.

Modern electron microscopes can access subnanometric spatial resolutions and thus are now able to image individual defects in nanostructures. However image interpretation is not straightforward and accurate structural models are still required for an in-depth understanding of defective atomic structures. In this context computational simulations represent a necessary complementary tool both for the interpretation of experimental data and for a deeper understanding of the specific physical and chemical properties at defective sites.

Classical density functional theory (DFT) methods have been shown to describe with a high accuracy the structure and electronics of defects in carbon materials. Point defects can be simulated using either clusters or periodic structures formed from hundred of atoms. However more extended defects such as extended dislocation lines $^{2}$, turbostratic or misaligned graphite ${ }^{3}$ or amorphous carbon ${ }^{4}$ require significantly larger models. Until recently density functional techniques have been limited to carbon models containing only few hundreds of atoms. This limitation is going to be overcome by very recent improvements in filtration techniques on Gaussian basis sets that can massively reduce computational time and memory requirements. $\frac{5}{-}$ Thanks to these methodological developments combined with the continuous speeding up of computing systems, routine study by full DFT of atomic structures with many thousands of atoms is becoming a realistic task.

However, beside ground state determination, the study of the dynamics of defective crystals remains too computationally intensive to tackle within the framework of current density functional theory. Examples of these specific problems include the growth mechanism of carbon nanostructures, their thermal or mechanical stability, the effects of structural reorganization induced by high energy particle irradiation, and the diffusion of extended defects such as vacancy clusters or dislocations. This range of problems are generally investigated using molecular dynamics or Monte Carlo techniques where forces and energies are evaluated using empirical or semi-empirical approaches $\frac{6,7}{7}$ However many dynamical problems in carbon nanostructures involve mechanisms of bond breaking and reconstruction that are usually poorly estimated by computational methods parametrized on ground state configurations.

The usage of density functional tight binding (DFTB) for the study of dynamics and reorganization of complex carbon structures represents an extremely helpful compromise between accuracy and speed $\Omega^{-10}$ Density functional tight binding parameters, in particular those derived for carbon, 10 are highly transferable, overcoming the main limitations of empirical and semi-empirical techniques at the reduced computational cost of standard tight binding. This is demonstrated by a wide range of problematics covered by a number of studies on carbon based systems. DFTB has been employed for instance in the study of the structure and energetics of point defects in single walled carbon nanotubes ${ }^{11-16}$ and more extended defective structures such as screw dislocations in multi walled carbon nanotubes ${ }^{17}$ and bonding between fullerenes and nanocones. 18 The capability of carbon DFTB parameters to reproduce complex rebydridization phenomena has been shown for example in the simulation of the thermal induced graphitization of nano-diamond surfaces,$\frac{19}{19}$ in monoatomic carbon chains 
formation at axial strain applied to carbon nanotubes, 20 and carbon nanostructure growth through $\mathrm{C}_{2}$ addition 21 DFTB molecular dynamics has been also employed in the study of self-assembling processes of fullerene cages $^{22-24}$ and nanotubes 25

In order to justify the use of DFTB approaches it is necessary to benchmark the results against more conventional approaches such as full DFT calculations. Surprisingly in the literature there is a lack of detailed comparative studies of this nature for defective carbon nanomaterials and for this reason we have undertaken the current work. We have first chosen a range of well characterised intrinsic point defect structures in graphene in order to benchmark the DFTB optimised geometries and formation energies. This is followed by a study of more complex unterminated and functionalised edges to quantify its capability with extended structural defects. As well as static ground state structures it is important to analyse the performance of the DFTB approach further from equilibrium and for this reason we next study defect diffusion and formation/annihilation barriers. Finally we take as a complex dynamic example the formation of points defects in carbon nanomaterials under the influence of an electron beam. As we show, DFTB is capable of remarkably accurate reproduction of full DFT calculations at a fraction of the computational cost, justifying its use in a wide range of structurally complex carbon nanoscale problems.

\section{COMPUTATIONAL METHOD}

DFT calculations are conducted using the AIMPRO2.0 $\operatorname{code}^{26,27}$ and the DFTB approximation as implemented in the dftb $+\operatorname{code}^{46}$, using comparable cells and k-point meshes. The AIMPRO calculations are performed under the local density approximation using a localised Gaussian basis set with 22 independent functions per carbon atom (12 per hydrogen and 40 per oxygen). Finite temperature Fermi smearing is used to control electron state population near to the Fermi level with temperature $\mathrm{kT}=0.04 \mathrm{eV}$. Spin polarised calculation have been performed for open shell configurations (carbon monovacancy, zigzag and Klein edges). Pseudopotentials are taken from Hartwingser-Goedecker-Hutter ${ }^{28}$ DFTB parameters have been derived by $\mathrm{M}$. Elstner et al 10 Both the DFT and DFTB calculations are fully self-consistent. No additional functionality such as Van der Waals corrections are used within the DFTB calculations.

\section{POINT AND LINE DEFECTS IN CARBON NANOSTRUCTURES}

In this section we present a comparative study on the structure and formation energies of topological defects in graphene and graphenic structures. We take as our first test system a series of standard intrinsic point de- fect structures in the graphene lattice, namely a single vacancy, a 5-8-5 divacancy pair, a "Stone-Wales" defect (a rotation of two carbon atoms through $90^{\circ}$ about their bond centre), and an "inverse Stone-Wales" defect (addition of a $\mathrm{C}_{2}$ pair to the graphene lattice across a single hexagon). Between them these defects contain a variety of local bonding including undercoordinated carbon atoms, dilated bonds, local out-of-plane distortions and combinations of resonant and localised single-/doublebonds. As such they represent a stringent test for DFTB. Through the use of an infinite graphene nanoribbon we then examine extended defects in the form of unterminated graphene sheet edges. These once again exhibit a range of bonding states including localised triple bond character (armchair and reconstructed zigzag edges), extended metallic states (zigzag edges) and singly coordinated carbon atoms (Klein edge).

In figure 1 we present structural models for the different types of point defects in graphene and several graphene edge configurations. In the figure we report the most notable bond lengths as obtained after optimization with DFT (blue values) and DFTB in its self consistent charge formulation (red values). The DFT results are detailed further in Ref. 29 .

The DFTB structures are in excellent agreement with the DFT data for all carbon coordinations, from the single coordination of a carbon atom at a Klein edge, through the double coordinate carbons at the zigzag and armchair edge or close to the vacancy site, to the modified triple coordinated states as in the Stone-Wales and inverse Stone-Wales defect $\left(\mathrm{C}_{2}\right.$ addition). All DFTBDFT bond lenght discrepancies are lower then $4 \%$ and most around $2 \%$.

Formation energies are presented in table $\llbracket$ and once again agreement is excellent, with DFTB point defect formation energies matching DFT values to within 1.5\% (and most less than 1\%). The largest error is in the Klein edge formation energy (19.7\%), which is understandable given that this is a physically unstable edge structure (repetition of the unit cell and breakage of the symmetry leads to spontaneous pairwise rebonding of the undercoordinated atoms ${ }^{29}$ ). The other edge formation energies deviate from the DFT values by $9.7 \%, 1.8 \%$ and $1.8 \%$ respectively.

Pristine graphene edges have dangling bonds at the edge atoms. Rehybridization (as considered above) or $\mathrm{H}$-termination is the simplest way to saturate these dangling bonds, while inducing only small edge strain. Edge functionalization by halogens or more complex functional groups (-OH or - $\mathrm{SH})$ has been shown to induce a significant strain along the ribbon edge, through steric hindrance, electrostatic repulsion between groups, intergroup bonding, etc. Being energetically unfavourable, this strain can be relieved via out-of-plane distortions. Specifically, hydroxyl $(-\mathrm{OH})$ terminated graphene nanoribbons of different widths have been shown to compensate the induced strain by forming a localised out-ofplane static ripple along the graphene sheet edge $\underline{30}$ A key 
Monovacancy

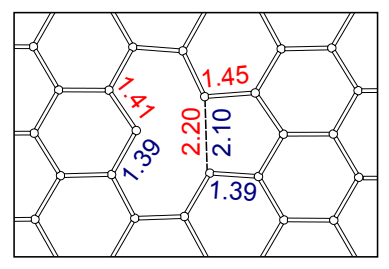

Armchair

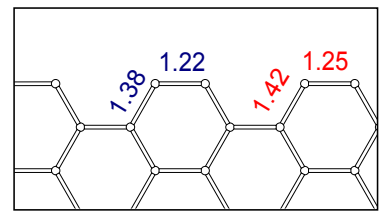

5-8-5 divacancy

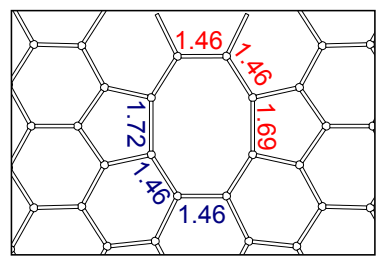

Zigzag

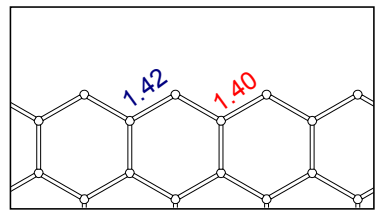

Stone-Wales

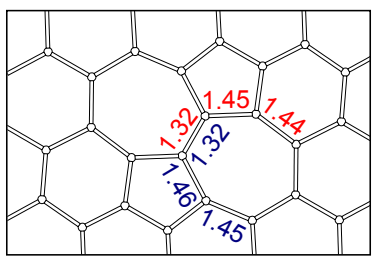

Klein

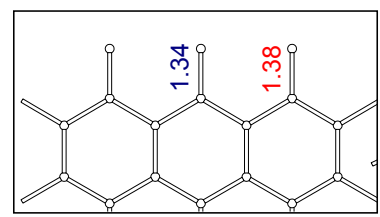

Inverse Stone Wales

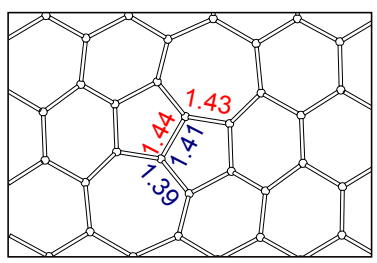

Reconstructed Zigzag

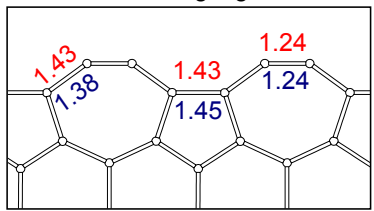

Figure 1: Structure of intrinsic point defects and unterminated edges in graphene. Values in the figures represent bond lengths in Angstroms obtained from DFT (blue) and DFTB (red). DFT values from Reference. ${ }^{29}$

\begin{tabular}{|c|c|c|}
\hline \multicolumn{3}{|l|}{ Point defects $(\mathrm{eV})$} \\
\hline & \multicolumn{2}{|c|}{ DFT DFTB } \\
\hline Mono-vacancy & 7.40 & 7.51 \\
\hline 5-8-5 Divacancy & 8.25 & 8.19 \\
\hline Stone-Wales & 4.86 & 4.85 \\
\hline Inverse Stone-Wales & 6.37 & 6.40 \\
\hline \multicolumn{3}{|l|}{$\begin{array}{ll}\text { Edges }(\mathrm{eV} / \AA)\end{array}$} \\
\hline & \multicolumn{2}{|c|}{ DFT DFTB } \\
\hline Zigzag edge & 1.34 & 1.21 \\
\hline Armchair edge & 1.10 & 1.08 \\
\hline Klein edge & 2.22 & 1.78 \\
\hline Zigzag reconstructed edge & 1.09 & 1.07 \\
\hline -OH terminated armchair ribbon & -2.26 & -3.53 \\
\hline
\end{tabular}

Table I: Formation energies for point defects and edges in graphene obtained by DFT and DFTB. DFT edge values from References 29,30 .

consequence of these functionalised nanoribbon edges is that both electronic and mechanical properties can be tuned 30

In figure 2 we present the structural model of an hydroxyl terminated graphene nanoribbon. Both DFT and DFTB calculations confirm that a rippled configuration is more stable than any flat structure, allowing the ribbon edge to relieve strain through out of plane distortion 30

Once again, bond lengths obtained with DFT and DFTB generally correlate well. The only discrepancy occurs at hydrogen bridges whose lengths are overestimated by DFTB. This bond dilation is also reflected in the edge formation energy in table 1 where the DFTB edge formation energy is too energetically stable compared to the DFT result. DFTB is known to tend towards overbinding for hydrogen-X bonds, and the dftb + code includes a damping correction to the short range contribution to the SCC interaction for hydrogen 31,32 which we did not use here. In addition we can also not exclude the effect of the limited size of the DFTB basis or the possibility of an incorrect estimation of the -OH group chemical potential, and further studies are needed to fully explain this difference.

In general we find a good correspondence in the energetics (formation energies) and structural characteristics obtained by DFTB with DFT results, for both intrinsic point and line defects in graphene, with reasonable structural and energetic agreement for heteroatom systems given the limitations discussed above. These results support the use of DFTB in future, notably for problems which can not be treated at the DFT level due to their size and complexity, or due to the necessity for long trajectories, for example DFTB-MD simulations on dynamics of graphene ribbon rippling (propagating and stationary waves along the edge).

\section{DEFECT DYNAMICS: FORMATION AND MIGRATION BARRIERS}

The description of complex dynamical phenomena such as the reorganization of carbon material when exposed to thermal treatments can be decomposed into several elementary processes such as atom and vacancy migration and nucleation, bond rotation, and carbon atom re-hybridization. Therefore, it is fundamental to derive accurately minimum energy reaction paths and activation energies of these elemental transformations in order to describe precisely more global transformations. However, the ability of carbon to re-hybridize can render even 


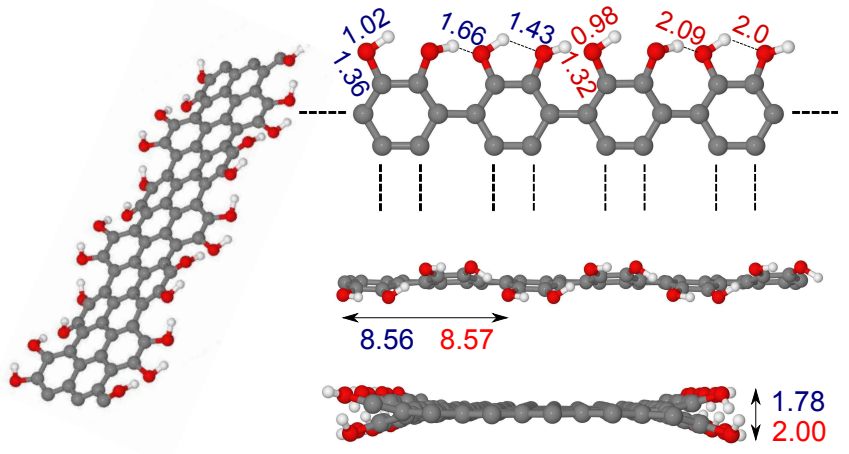

Figure 2: Structure of -OH terminated armchair graphene nanoribbon where values in the figures represent bond length (in $\AA$ ) obtained from DFT (blue) and DFTB (red). DFT values from Reference ${ }^{30}$.

\begin{tabular}{lcc}
\hline \hline & \multicolumn{2}{c}{ Barrier (eV) } \\
\hline & DFT & DFTB \\
\hline Vacancy Migration & $1.37^{37}$ & 1.29 \\
Stone-Wales Formation & $9.2^{38}$ & 10.4 \\
Stone-Wales Annihilation & $4.4^{38}$ & 4.7 \\
\hline \hline
\end{tabular}

Table II: Calculated barriers $(\mathrm{eV})$ for point defect formation and migration in graphene obtained by DFT and DFTB using the nudged elastic band method.

elemental reaction paths highly complicated, often introducing intermediate metastable configurations.

The nudged elastic band (NEB) $\operatorname{method}^{33,34}$ is a useful techniques for exploring in an efficient and automatic way a large region of the configuration space and derive complex minimum energy paths. However, a large number of intermediate images is generally required for obtaining with a good accuracy the saddle point configuration and associated activation energies. Furthemore the NEB method converges usually only after a large number of optimization steps. The high computational cost of the NEB technique represents a strong limit for its usage in the framework of high level computational approaches.

A good compromise can be obtained using the density functional tight binding theory (DFTB). A combined NEB-DFTB approach has already been shown to give results comparable to other higher-end techniques ${ }^{35}$. In the context of layered materials, DFTB-NEB has been successfully employed in the study of single vacancies and vacancy complex migration in boron nitride monolayers. 36 Here we compare the possibilities of a DFTB-NEB approach with equivalent more time consuming DFT-NEB calculations for the study of topological transformations in carbon. Two example are presented where bond breaking occurs: mono-vacancy migration and Stone-Wales bond rotation in a graphene plane.

Mono-vacancy diffusion in graphene occurs when a doubly coordinated carbon close to a vacancy site breaks its two covalent bonds for rebonding with the opposite atom pair neighboring the vacancy (the complete migration path also involves a bonding rearrangement around the vacancy core but this has a very low activation barrier). The minimum energy path saddle point obtained by DFTB-NEB corresponds to a configuration where the migrating carbon atom lies in the middle point between its initial and final position. The graphene sheet undergoes a slight asymmetric out-of-plane deformation allowing the migrating atom to locate itself at the center of a compressed tetrahedron. The DFTB activation energy we obtain is $1.29 \mathrm{eV}$, which is in an extremely good agreement with the value of $1.37 \mathrm{eV}$ obtained by an analogous DFT-NEB study ${ }^{37}$ ( $6 \%$ underestimation).

The 90 degree bond rotation required for the formation of a Stone-Wales defect involves the breaking and reconstruction of two covalent bonds. Using DFTB-NEB we obtain an activation energy for defect formation of $10.4 \mathrm{eV}$, with the corresponding annihilation barrier for the reverse reaction of $4.7 \mathrm{eV}$. These compare extremely well to equivalent DFT values of $9.2 \mathrm{eV}$ and $4.4 \mathrm{eV}$ respectively ${ }^{38}$, with DFTB thus overestimating the DFT values by 13 and $7 \%$ respectively.

These activation barrier calculations represent a stringent test for DFTB, passing through structures which are far from the equilibrium defect ground states. The excellent agreement between DFT and DFTB justifies the use of DFTB in calculations of dynamic systems, and in the following section we show an example of this where we use DFTB to examine atom loss during electron irradiation of carbon nanostructures.

\section{DEFECT DYNAMICS: ELECTRON IRRADIATION IN CARBON NANOSTRUCTURES}

Electron irradiation is an unavoidable and generally unwanted side effect when high energy electrons are used for imaging and analysis of nanostructures, such as in transmission electron microscopy (TEM), but it can also be used to deliberately restructure a carbon nanosystem in order to tune its mechanical and electronic properties. In this context it is desirable to be able to finely describe the probability that a specific structural transformation occurs under electron irradiation and how this probability depends on the energy of the electron beam.

Electron irradiation effects in carbon materials can be explained mostly by direct elastic scattering between the relativistic electrons of the beam and the atomic nucleus. For a given electron beam energy atoms can only be sputtered along directions for which the transfered energy is above a certain emission direction dependent energy threshold.

An analytic expression for the differential cross section as a function of the emission direction has been derived by $\operatorname{Mott}^{39}$ and a useful approximation of the original expression has been obtained by McKinsley and Feshbach. $\underline{\underline{40}}$ Total emission cross sections are derived by 
integrating the differential cross section over the solid angle defined by the possible emission directions at a given electron beam energy.

In Ref $\stackrel{41}{ }$ we proposed a methodology for deriving anisotropic emission energy threshold maps using extended molecular dynamics simulations. The procedure consists in imparting an initial momentum to the atom to sputter (direction and speed). The system is successively allowed to evolve in a microcanonical ensemble and at the end of the simulation the final position of the atom is analyzed. The MD simulation is repeated increasing progressively the initial speed, up to the critical limit for which the atom is ejected. The procedure is reiterated for a number of different emission directions.

To obtain accurate energy threshold maps a high number of directions have to be considered and the step size used to increase the initial velocity should be sufficiently small. In the case of perfect graphene a map is obtained performing about 10000 molecular dynamics calculations, each of $200 \mathrm{MD}$ steps on a structure containing 200 atoms. This number of calculations is too computationally demanding for standard density functional techniques, but DFTB can produce a full emission map at an affordable computational cost. This techniques has thus be successfully used in the study of sputtering in perfect and defective graphene and monolayer boron-nitride as well as in the study of irradiation induced bond rotations in graphene ${ }^{41-44}$.

In figure 3 we present the DFTB-MD derived emission energy threshold map for a carbon atom from a graphene plaine. DFTB estimates the minimum ejection energy to be around $23 \mathrm{eV}$, corresponding to an emission direction orthogonal to the plane. A recent work has compared the DFT and DFTB emission energies obtaining, in the DFT case, a value of $22.2 \mathrm{eV}{ }^{43}$ The excellent agreement between the DFT and DFTB values (less than $4 \%$ difference) validates the use of DFTB for sputtering simulations in carbon materials. However in the case of boron and nitrogen sputtering from a BN plane the DFTB values are lower than the DFT. This discrepancy has been discussed by the author as an inadequate description of charge transfer in DFTB calculations for the $\mathrm{BN}$ system. 43

Considering the kinematic of the scattering problem carbon atoms can be sputtered from a graphene plane by electrons with an energy above $113 \mathrm{keV}$. Experimental TEM studies find an electron beam energy limit situated between 90 and $100 \mathrm{keV}$. This discrepancy can be reasonably attributed to the well known dissociation energy overestimation occurring in DFT-LDA that also affects the DFTB parameters. This error can be considered as systematic and the theoretical results be can corrected by recalibrating on the experimental values.

In Fig. 4 we present the total knock-on cross section as a function of the electron beam energy for a carbon atom in a graphene plane. A commonly used approximation considers the emission energy threshold as independent of the emission direction, an analytic expression for the

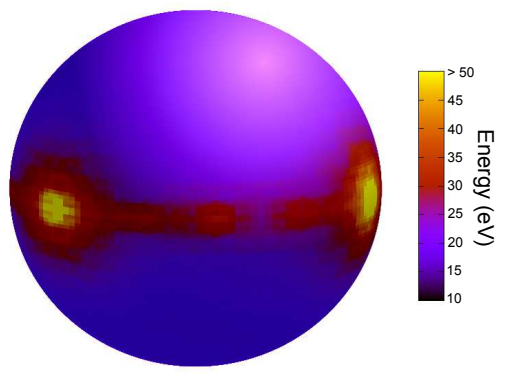

Figure 3: Emission energy threshold map for a carbon atom in a graphene plane. The spherical coordinate represent the emission direction and the color scale the minimum emission kinetic energy. The equatorial plane corresponds to emission directions on the graphene sheet, poles to directions orthogonal to the sheet.

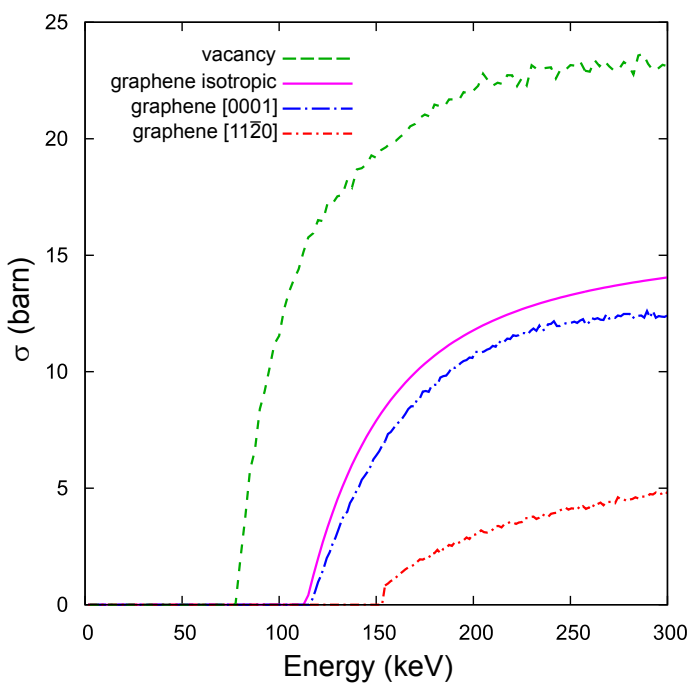

Figure 4: Sputtering cross sections for a carbon atom in a graphene plane using the isotropic emission threshold approximation and the anisotropic approximation when the electron beam direction is orthogonal or parallel to the plane. Sputtering cross sections for a carbon atom neighbouring a preexisting vacancy in graphene (beam direction orthogonal)

total cross section can then be found. $\stackrel{45}{ }$ This assumption brings however, as shown in Fig. 4 to overestimate the integration region and thus the emission cross sections and it cannot take into account the strong variation of cross section as a function of the electron beam orientation in respect to the graphene. In figure 4 we present also the cross section for a carbon atom neighboring a pre-existing vacancy site. The reduced coordination of the knocked carbon atom makes sputtering more probable than for an atom in perfect graphene. This higher cross section explains the vacancy clustering in graphene observed by TEM.

Calculated cross sections have been used to optimize irradiation conditions in a scanning transmission electron microscope for reshape individual single walled carbon

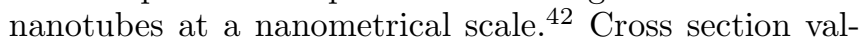


ues can also be used in kinetic Monte Carlo simulations of the global transformation of carbon structures under electron irradiation: calculated cross sections attribute a sputtering probability to each carbon atom, DFTB can successively be employed to optimize the system after each ejection event.

\section{CONCLUSIONS}

The versatility of carbon in its range of bonding leads to a rich variety of low symmetry materials, structures and defects. However the corresponding size of the resultant calculations, the range of minima to explore, and the complex energy surfaces in non-equilibrium situations render many such problems outside the scope of conventional density functional approaches. We show in the current study that the density functional tight binding approach is able to successfully reproduce, with high quantitative accuracy, both structural and energetic data from full density functional calculations at a fraction of the computational cost. Calculations of atom knock-on cross sections under electron irradiation provide an example where DFTB calculations are able to advance and guide our manipulation of carbon materials at the atomic scale. These results confirm that DFTB represents a powerful tool for computationally intensive studies of carbon nanomaterials.

\section{Acknowledgments}

We would like to thank R. Barthel for the implementation of the NEB technique using DFTB, and B. Hourahine for useful discussions. CPE, VI and PW acknowledge French ANR P3N project ANR-09-NANO016-04 "Nanosim_Graphene" for funding. All authors thank COST project MP0901 "NanoTP" for support.
* Electronic address: alberto.zobelli@u-psud.fr

1 I. Suarez-Martinez, N. Grobert, and C. Ewels, Carbon p. In Press (2011), doi:10.1016/j.carbon.2011.11.002.

2 R. H. Telling and M. I. Heggie, Philosophical Magazine Letters 83(7), 411 (2003).

${ }^{3}$ J. M. Campanera, G. Savini, I. Suarez-Martinez, and M. I. Heggie, Phys. Rev. B 75(Jun), 235449 (2007).

4 J. Robertson, Advances in Physics 35(4), 317 (1986).

${ }^{5}$ M. J. Rayson and P. R. Briddon, Phys. Rev. B 80, 205104 (2009).

${ }^{6}$ S. Irle, Y. Ohta, Y. Okamoto, A. Page, Y. Wang, and K. Morokuma, Nano Research 2(10), 755-767 (2009).

7 T. Petersen, I. Snook, I. Yarovsky, and D. McCulloch, Physical Review B 72(12), 125417 (2005).

8 D. Porezag, T. Frauenheim, T. Köhler, G. Seifert, and R. Kaschner, Phys. Rev. B 51, 12947 (1995).

9 G. Seifert, D. Porezag, and T. Frauenheim, Int. J. Quantum Chem. 58, 185 (1996).

10 M. Elstner, D. Porezag, G. Jungnickel, J. Elsner, M. Haugk, T. Frauenheim, S. Suhai, and G. Seifert, Phys. Rev. B 58, 7260 (1998).

11 J. Kotakoski, A. Krasheninnikov, and K. Nordlund, Phys. Rev. B 74(24), 245420 (2006).

12 M. Sternberg, L. Curtiss, D. Gruen, G. Kedziora, D. Horner, P. Redfern, and P. Zapol, Phys. Rev. Lett. 96(7), 75506 (2006).

13 S. Malola, H. Häkkinen, and P. Koskinen, Phys. Rev. B 81(16), 165447 (2010).

14 A. V. Krasheninnikov, K. Nordlund, P. O. Lehtinen, A. S. Foster, A. Ayuela, and R. M. Nieminen, Carbon 42(5-6), 1021 (2004).

15 E. Cruz-Silva, A. Botello-Méndez, Z. Barnett, X. Jia, M. Dresselhaus, H. Terrones, M. Terrones, B. Sumpter, and V. Meunier, Phys. Rev. Lett. 105(4), 45501 (2010).

16 A. V. Krasheninnikov, P. O. Lehtinen, A.S. Foster, and R. M. Nieminen, Chem. Phys. Lett. 418(1-3), 132 (2006).

17 I. Suarez-Martinez, G. Savini, A. Zobelli, and M. Heggie, J. Nanosci. and Nanotechnol. 7(10), 3417 (2007).
18 I. Suarez-Martinez, M. Monthioux, and C.P. Ewels, J. Nanosci. Nanotech. 9, 6144 (2009).

19 V. Ivanovskaya and A. Ivanovskii, Inorganic Materials 43, 349 (2007).

${ }^{20}$ V. Ivanovskaya, N. Ranjan, T. Heine, G. Merino, and G. Seifert, Small 1(4), 399 (2005).

21 C. P. Ewels, G. Van Lier, P. Geerlings, and J. C. Charlier, J. Chem. Inf. Model. 47(6), 2208 (2007).

22 B. Saha, S. Shindo, S. Irle, and K. Morokuma, ACS Nano 3(8), 2241 (2009).

23 S. Irle, G. Zheng, Z. Wang, and K. Morokuma, The Journal of Physical Chemistry B 110(30), 14531 (2006).

${ }^{24}$ S. Irle, G. Zheng, M. Elstner, and K. Morokuma, Nano Letters 3(4), 465 (2003).

25 Y. Ohta, Y. Okamoto, S. Irle, and K. Morokuma, ACS Nano 2(7), 1437 (2008).

26 R. Jones and P. Briddon, Semicond. Semimetals 51A, 287 (1998).

27 M. Rayson and P. Briddon, Comp. Phys. Comm. 178, 128 (2008).

28 C. Hartwigsen, S. Goedecker, and J. Hütter, Phys. Rev. B. 58, 3641 (1998).

29 V. V. Ivanovskaya, A. Zobelli, P. Wagner, M. I. Heggie, P. R. Briddon, M. J. Rayson, and C. P. Ewels, Phys. Rev. Lett. 107, 065502 (2011).

30 P. Wagner, C. P. Ewels, V. V. Ivanovskaya, P. R. Briddon, A. Pateau, and B. Humbert, Phys. Rev. B 84, 134110 (2011).

31 M. Gaus, M. Cui, and J. Elstner, J. Chem. Theory Comput. 7, 931-948 (2011).

32 Y. Yang, H. Yu, D. York, Q. Cui, and J. Elstner, J. Phys. Chem. A 111, 10861 (2007).

${ }^{33}$ G. Mills and H. Jónsson, Phys. Rev. Lett. 72, 1124 (1994).

${ }^{34}$ G. Henkelman, B. Uberuaga, and H. Jónnson, J. Chem. Phys. 113, 9901 (2000).

35 G. Fischer, R. Barthel, and G. Seifert, Eur. Phys. J. D 35, 479 (2005).

36 A. Zobelli, C. P. Ewels, A. Gloter, and G. Seifert, Phys. Rev. B 75, 094104 (2007). 
37 H. Zhang, M. Zhao, X. Yang, H. Xia, X. Liu, and Y. Xia, Diam. Relat. Mater. 19(10), 1240-1244 (2010).

${ }^{38}$ L. Li, S. Reich, and J. Robertson, Phys. Rev. B 72, 184109 (2005).

39 N. Mott, Proc. Roy. Soc. A 135, 429 (1932).

40 W. McKinsley and H. Feshbach, Phys. Rev. 74, 1759 (1948).

41 A. Zobelli, A. Gloter, C. P. Ewels, G. Seifert, and C. Colliex, Phys. Rev. B 75, 245402 (2007).

42 A. Zobelli, A. Gloter, C. P. Ewels, and C. Colliex, Phys. Rev. B 77, 045410 (2008).
43 J. Kotakoski, C. Jin, O. Lehtinen, K. Suenaga, and A. Krasheninnikov, Physical Review B 82(11), 113404 (2010).

44 J. Kotakoski, J. C. Meyer, S. Kurasch, D. Santos-Cottin, U. Kaiser, and A. V. Krasheninnikov, Phys. Rev. B 83, 245420 (2011).

45 F. Banhart, Rep. Prog. Phys. 62, 1181 (1999).

46 http://www.dftb-plus.info 